\title{
A Systematic Review of Classical Entropy Peng $\mathrm{Li}^{1,2}$ a , Gang Su ${ }^{1,2}$ and Xiao-Ping Qin ${ }^{1,2}$
}

\author{
${ }^{1}$ School of Mathematics, Physics and Information Science, Zhejiang Ocean University, Zhoushan, \\ Zhejiang 316022, China \\ ${ }^{2}$ Key Laboratory of Oceanographic Big Data Mining \& Application of Zhejiang Province, Zhoushan, \\ Zhejiang 316022, China \\ apengli.physics@qq.com
}

Keywords: Entropy, State Variable, Thermodynamical Potential.

Abstract. It is difficult to grasp classical entropy because of the apparent complexity of relevant mathematics. Here we first give some physical thoughts on the concept of entropy, then examine its mathematical structrure. Several universal results are given by a set of equations governing the partial derivatives of internal energy and enthalpy, and applications are discussed with real gas models. This paper provides a systematic treatment of entropy beyond the ideal gas and may help understanding the foundation of thermodynamics.

\section{Introduction}

In classical thermodynamicsm [1,2], the entropy of a system is defined by an integral of heat over temperature along a reversible trajectory on a phase plane spanned by independent state variables. Only if the integral does not depend on trajectory can entropy be regarded as a state variable, i.e., a scalar function of other state variables. Under such condition entropy is called a thermodynamic potential and the whole theory of classical thermodynamics is built on such ground.

Among all the state variables, pressure $P$ and volume $V$ are mechanical quantities and can be directly measured from the state. Temperature $T$ is not a mechanical quantity. However, it can be defined as a scalar function of $P$ and $V$ via the state equation of an ideal gas. Assuming all systems in equilibrium with an ideal gas acquire the same temperature, $T$ can be safely regarded as a state variable in genral. In other words, $P, V$, and $T$ are explicitly defined by the state, not by an integral, it is mathematically redundant to verify their validity as state variables. Furthermore, internal energy $U$ is introduced to account for the overall energy of a system participating the thermodynamic processes. It is indeed an axiom that such energy depends only on the state but not on the trajectory, otherwise energy cannot be conserved. It is the first law of thermodynamics asserts that $U$ must be a state variable. Given $U$ a state variable, it is also valid to define enthalpy $H=U+P V$ as a state variable as well.

To explain that entropy is a state variable is more complicated. We should best start with the Carnot's gedanken experiment [3]. For all reversible heat engines working between a hot reservoir at temperatures $T_{1}$ and a cold reservoir at temperature $T_{2}$, Carnot suggests that they must have the same efficiency $\zeta$, which is merely a function of $T_{1}$ and $T_{2}$. Because otherwise a second-type perpetual motion machine can be constructed by using two such engines in tandem, a forward-running one drives a backward-running one, and the net effect is to extract heat from one of the reservoirs and convert it completely into work, which obviously violates the second law of thermodynamics. Furthermore, it can be strictly proved that $\zeta=1-T_{2} / T_{1}$, provided the engine is implemented by ideal gas. In other words, we always have $\Delta Q_{1} / T_{1}+\Delta Q_{2} / T_{2}=0$, which indicates that entropty $\Delta Q / T$ is a conserved quantity in a full cycle. Since any reversible process can be decomposed as a series of Carnot's cycle, the entropy of ideal gas is then conserved for arbitrary closed reversible trajectory. Moreover, because reversible heat engines implemented by any substances should have the same efficiency $\zeta$, the above reasoning for entropy conservation applies for any real substances. In other words, entropy can be regarded as a state variable in any reversible processes.

The above discussion of Carnot efficiency $\zeta$ is built on two special properties of ideal gas: (i) the state equation $P V=n R T$, and (ii) that the internal energy $U$ is solely determined by temperature $T$. The 
latter is based on a kinetic theory which is independent of the second law of thermodynamics. As a reward, knowing entropy as a universal state variable provides a useful knowledge on the internal energy of any substance. This may help to unveil the microscopic nature of the substance. In this paper we derive a set of such equations governing the behavior of internal energy and enthalpy, and discuss their applications to several real gas models.

\section{Theory}

The first law of thermodynamics asserts that

$$
\delta Q=d U+P d V
$$

where $\delta$ is used where the differential is not exact. The classical definition of entropy reads

$$
d S=\frac{\delta Q}{T}=\frac{1}{T}(d U+P d V)
$$

where $U$ can be expressed as a function of two independent variables chosen from $P, V$, and $T$. Next we expand the above equation in three approaches as below:

V-T plane. In this case $U=U(V, T)$ and thus

$$
d U=\left(\frac{\partial U}{\partial V}\right)_{T} d V+\left(\frac{\partial U}{\partial T}\right)_{V} d T .
$$

Substitute it back to equation (2) results in

$$
d S=f_{V}(V, T) d V+f_{T}(V, T) d T,
$$

where

$$
f_{V}(V, T)=\frac{1}{T}\left[\left(\frac{\partial U}{\partial V}\right)_{T}+P\right], f_{T}(V, T)=\frac{1}{T}\left(\frac{\partial U}{\partial T}\right)_{V} .
$$

The integral of $d S$ is trajectory independent only if the vector field $\left(f_{V}, f_{T}\right)$ is rotationless [4] in the V-T plane, i.e.,

$$
\left(\frac{\partial f_{V}}{\partial T}\right)_{V}=\left(\frac{\partial f_{T}}{\partial V}\right)_{T}
$$

which finally yields

$$
\left(\frac{\partial U}{\partial V}\right)_{T}=T\left(\frac{\partial P}{\partial T}\right)_{V}-P .
$$

This equation describes how internal energy varies with volume at a constant temperature. Furthermore, by a combined application of the cyclic, reciprocal, and chain rules for partial derivatives [1], the above equation can be recast into

$$
\eta_{t}=\frac{1}{c_{v}}\left(P-\frac{\alpha}{\kappa} T\right)
$$

where 


$$
\eta_{t} \equiv\left(\frac{\partial T}{\partial V}\right)_{U}, c_{v} \equiv\left(\frac{\partial U}{\partial T}\right)_{V}, \kappa \equiv-\frac{1}{V}\left(\frac{\partial V}{\partial P}\right)_{T}, \text { and } \alpha \equiv \frac{1}{V}\left(\frac{\partial V}{\partial T}\right)_{P},
$$

are the Joule coefficient of temperature for free expansion, the isochoric heat capacity, the isothermal compressibility and the isobaric expansivity, respectively. Note that equation (8) is constructed via experimentally measurable parameters.

P-T plane. In this case it is more convenient to express the first law [eq. (1)] via enthalpy as

$$
\delta Q=d H-V d P
$$

and expand $d H$ as

$$
d H=\left(\frac{\partial H}{\partial P}\right)_{T} d P+\left(\frac{\partial H}{\partial T}\right)_{P} d T
$$

Then we obtain

$$
d S=\left[\frac{1}{T}\left(\frac{\partial H}{\partial P}\right)_{T}-\frac{V}{T}\right] d P+\frac{1}{T}\left(\frac{\partial H}{\partial T}\right)_{P} d T .
$$

Similarly, the trajectory independence of entropy requires that

$$
\frac{\partial}{\partial T}\left[\frac{1}{T}\left(\frac{\partial H}{\partial P}\right)_{T}-\frac{V}{T}\right]_{P}=\frac{\partial}{\partial P}\left[\frac{1}{T}\left(\frac{\partial H}{\partial T}\right)_{P}\right]_{T},
$$

which finally yields

$$
\left(\frac{\partial H}{\partial P}\right)_{T}=V-T\left(\frac{\partial V}{\partial T}\right)_{P} .
$$

This equation describes how enthalpy varies with pressure at a constant temperature, and can be cast into an experimentally verifiable form as

$$
\mu=\frac{V}{c_{p}}(\alpha T-1),
$$

where

$$
\mu \equiv\left(\frac{\partial T}{\partial P}\right)_{H}, \text { and } c_{v} \equiv\left(\frac{\partial U}{\partial T}\right)_{V}
$$

are the Joule-Thomson coefficient for isoenthalpic expansion, and the isobaric heat capacity, respectively.

V-P plane. In this case we expand $d U$ as

$$
d U=\left(\frac{\partial U}{\partial V}\right)_{P} d V+\left(\frac{\partial U}{\partial P}\right)_{V} d P
$$

Substitute it into equation (2) we find 


$$
d S=\left[\frac{1}{T}\left(\frac{\partial U}{\partial V}\right)_{P}+\frac{P}{T}\right] d V+\frac{1}{T}\left(\frac{\partial U}{\partial P}\right)_{V} d P
$$

And the trajectory independence of entropy again requires

$$
\frac{\partial}{\partial P}\left[\frac{1}{T}\left(\frac{\partial U}{\partial V}\right)_{P}+\frac{P}{T}\right]_{V}=\frac{\partial}{\partial V}\left[\frac{1}{T}\left(\frac{\partial U}{\partial P}\right)_{V}\right]_{P},
$$

which leads to

$$
\left(\frac{\partial T}{\partial P}\right)_{V}\left[\left(\frac{\partial U}{\partial V}\right)_{P}+P\right]-\left(\frac{\partial T}{\partial V}\right)_{P}\left(\frac{\partial U}{\partial P}\right)_{V}=T
$$

By multiplying $(\partial P / \partial V)_{T}$ to both side and applying equation (7) we finally obtain

$$
\left(\frac{\partial U}{\partial V}\right)_{P}-\left(\frac{\partial U}{\partial V}\right)_{T}=\left(\frac{\partial T}{\partial V}\right)_{P}\left(\frac{\partial U}{\partial T}\right)_{V} .
$$

This equation describes how the partial derivative of internal energy with respect to volume differs under isobaric and isothermal processes. Meanwhile, it can be recast with measurable parameters into

$$
\eta_{t} \alpha-\eta_{p} \kappa=\frac{1}{V}
$$

where

$$
\eta_{p}=\left(\frac{\partial P}{\partial V}\right)_{U}
$$

is the Joule coefficient of pressure in free expansion.

Equations (7), (14) and (21) are the key consequences brought by the assertion that entropy is a state variable. They set a universal constriction for all substances on how their internal energy is related with other state variables. Such constriction is intrinsically required by the mathematical structure of classical thermodynamics. As a reward the equations help to draw a quantitative clue of the underlying microscopic interaction, which dictates the form of internal energy. Equations (8), (15) and (22), as expressed by measurable parameters, provide an opportunity to test the validity of thermodynamics for arbitrary substance. In the next section we apply the findings on both ideal and real gases.

\section{Applications}

Ideal gas. An ideal gas (of one mole) abides a state equation $P V=R T$, and accordingly

$$
\left(\frac{\partial P}{\partial T}\right)_{V}=\frac{R}{V} \text {, and }\left(\frac{\partial V}{\partial T}\right)_{P}=\frac{R}{P} .
$$

Substitute the above equations to equations (7) and (14) we obtain

$$
\left(\frac{\partial U}{\partial V}\right)_{T}=0, \text { and }\left(\frac{\partial H}{\partial P}\right)_{T}=0 .
$$


The results indicate that both $U$ and $H$ depends solely on temperature $T$. It is consistent with the kinetic theory of an ideal gas. Moreover, the Joule and Joule-Thomson coefficients are found to be

$$
\eta_{t}=0, \eta_{p}=\frac{P}{V}, \text { and } \mu=0,
$$

which are also consistent with experiment.

Real gases. We start by looking at the Van der Waals model [1] with a state equation

$$
\left(P+\frac{a}{V^{2}}\right)(V-b)=R T,
$$

and accordingly find

$$
\left(\frac{\partial P}{\partial T}\right)_{V}=\frac{R}{V-b} .
$$

Substitute it into equation (7) we arrive at

$$
\left(\frac{\partial U}{\partial V}\right)_{T}=\frac{a}{V^{2}} .
$$

This indicates that when temperature, i.e., the kinetic energy of the molecules, is held constant, the internal energy of a Van der Waals gas increases as volume expands. It can be explained only if there exists an attractive interaction among gas molecules, therefore potential energy increases as volume expands. Such increment in potential energy scales as $1 / V^{2}$, which tends to vanish as the gas become increasingly dilute, therefore is asymptotically consistent with the ideal gas.

For a Berthelot model [1] the state equation reads

$$
\left(P+\frac{a^{\prime}}{T V^{2}}\right)(V-b)=R T,
$$

and it is not difficult to show that

$$
\left(\frac{\partial U}{\partial V}\right)_{T}=\frac{2 a^{\prime}}{T V^{2}}
$$

Now besides the general attractive molecular interaction, an additional temperature dependence manifest itself via the $1 / T$ factor. The potential energy increment is less significant at high temperatures. This can be qualitatively explained via molecular collision. At higher temperatures more collisions occur, therefore molecules have more chance to hold a small intermolecular distance where potential energy is lowered. This secondary effect cancels part of the energy increase at expansion.

As a more accurate model, the Redlich-Kwong equation [1] is shown below

$$
\left(P+\frac{a^{\prime \prime}}{T^{1 / 2} V(V+b)}\right)(V-b)=R T,
$$

which finally yields 


$$
\left(\frac{\partial U}{\partial V}\right)_{T}=\frac{3 a^{\prime \prime}}{2 T^{1 / 2} V(V+b)}
$$

Now the finite volume of each individual molecule plays a similar role as temperature. For a larger $b$ leads to a higher collision rate, therefore effectively reduces the potential energy.

Moreover, substitute equations (29), (31) and (33) into equation (9) and use

$$
\left(\frac{\partial T}{\partial V}\right)_{U}=-\frac{1}{c_{v}}\left(\frac{\partial U}{\partial V}\right)_{T}
$$

we can find that the Joule coefficients for the Van der Walls, the Berthelot, and the Redlich-Kwong gases are given by

$$
\eta_{t}=-\frac{1}{c_{v}} \frac{a}{V^{2}}, \quad \eta_{t}{ }^{\prime}=-\frac{1}{c_{v}} \frac{2 a^{\prime}}{T V^{2}}, \text { and } \eta_{t}{ }^{\prime \prime}=-\frac{1}{c_{v}} \frac{3 a^{\prime}}{2 T^{1 / 2} V(V+b)} .
$$

This means the Joule's effect is genrally proportional to the strength of the intermolecular interaction, and decreases with respect to volume. The temperature dependence for the latter two models can be explained via the same reasoning as discussed for equation (31).

\section{Summary}

In this paper we discussed the mathematical formalism of classical entropy. In thermodynamics entropy as a state variable is essentially an axiom developed from Carnot's gedanken experiment. The mathematical consequences brought by this point are shown systematically. Provided with a phenomenological state equation, these results help to understand the physical nature of the underlying molecular interaction. On the other hand, the validity of classical thermodynamics can thus be tested via experimentally measurable parameters.

\section{Acknowledgements}

This work was supported by the Teaching Enhancement Program of Zhejiang Ocean University and NSFC Grant No. 11305141, 11104248, 11204271 and 11304281.

\section{References}

[1] A.H. Carter: Classical and Statistical Thermodynamics (Pearson Education, Prentice-Hall, 2001)

[2] F.C. Andrews: Thermodynamics: Principles and Applications (John Wiley \& Sons, London, 1971)

[3] D.V. Schroeder: An Introduction to Thermal Physics (Addison Wesley Longman, New York, 2000)

[4] D.J. Griffiths: Introduction to Electrodynamics (Pearson Education, Prentice-Hall, 2005) 